\title{
Periostin is essential for cardiac healing after acute myocardial infarction
}

\author{
Masashi Shimazaki, ${ }^{1}$ Kazuto Nakamura, ${ }^{2}$ Isao Kii, ${ }^{1}$ Takeshi Kashima, ${ }^{3}$ \\ Norio Amizuka, ${ }^{4}$ Minqi Li, ${ }^{4}$ Mitsuru Saito, ${ }^{5}$ Keiichi Fukuda, ${ }^{6}$ \\ Takashi Nishiyama, ${ }^{1}$ Satoshi Kitajima, ${ }^{7}$ Yumiko Saga, ${ }^{8}$ Masashi Fukayama, ${ }^{3}$ \\ Masataka Sata, ${ }^{2}$ and Akira Kudo ${ }^{1}$
}

\footnotetext{
'Department of Biological Information, Tokyo Institute of Technology, Yokohama 226-8501, Japan

2Department of Cardiovascular Medicine and ${ }^{3}$ Department of Pathology, Graduate School of Medicine, The University of Tokyo, Tokyo 113-0033, Japan

${ }^{4}$ Center for Transdisciplinary Research, Niigata University, Niigata 951-8514, Japan

${ }^{5}$ Department of Orthopaedic Surgery, Jikei University School of Medicine, Tokyo 105-8461, Japan

${ }^{6}$ Department of Regenerative Medicine and Advanced Cardiac Therapeutics, Keio University School of Medicine,

Tokyo 160-8582, Japan

${ }^{7}$ Division of Cellular and Molecular Toxicology, National Institute of Health Science, Tokyo 158-8501, Japan

${ }^{8}$ Division of Mammalian Development, National Institute of Genetics, Mishima 411-8540, Japan
}

\section{CORRESPONDENCE}

Akira Kudo:

akudo@bio.titech.ac.jp

Acute myocardial infarction (AMI) is a common and lethal heart disease, and the recruitment of fibroblastic cells to the infarct region is essential for the cardiac healing process. Although stiffness of the extracellular matrix in the infarct myocardium is associated with cardiac healing, the molecular mechanism of cardiac healing is not fully understood. We show that periostin, which is a matricellular protein, is important for the cardiac healing process after AMI. The expression of periostin protein was abundant in the infarct border of human and mouse hearts with AMI. We generated periostin ${ }^{-1-}$ mice and found no morphologically abnormal cardiomyocyte phenotypes; however, after AMI, cardiac healing was impaired in these mice, resulting in cardiac rupture as a consequence of reduced myocardial stiffness caused by a reduced number of $\alpha$ smooth muscle actin-positive cells, impaired collagen fibril formation, and decreased phosphorylation of FAK. These phenotypes were rescued by gene transfer of a spliced form of periostin. Moreover, the inhibition of FAK or $\alpha v$-integrin, which blocked the periostin-promoted cell migration, revealed that $\alpha v$-integrin, $F A K$, and Akt are involved in periostin signaling. Our novel findings show the effects of periostin on recruitment of activated fibroblasts through FAK-integrin signaling and on their collagen fibril formation specific to healing after AMI.

Periostin, which is an extracellular matrix (ECM) molecule of the fasciclin family, acts in cell adhesion, migration, and growth in vitro (1-6). In the heart, periostin is expressed at very early stages of embryogenesis; however, it is not detected in the normal adult myocardium, except in the valves $(7,8)$ and in the case of various heart diseases (9-12).

The early cardiac healing process after acute myocardial infarction (AMI) can be divided into two successive phases: the inflammatory phase and the scar formation phase. In the inflammatory phase, monocytes and lymphocytes infiltrate into the necrotic myocardium, whereas in the scar formation phase, activated interstitial or circulating fibroblasts increase their motility

The online version of this article contains supplemental material and migrate into the lesion. The activation of TGF $\beta$ is important for regulation of this latter process. Myofibroblasts expressing $\alpha$ smooth muscle actin ( $\alpha$ SMA) induced by TGF $\beta$ are specialized fibroblasts that share characteristics with smooth muscle cells (SMCs). They play an important role in wound healing by synthesizing ECM and exerting strong contraction forces to minimize wound areas (13-16). Regarding the inflammatory phase, recent knockout mouse studies indicated a positive association of inflammatory factors with cardiac rupture or dilation (17-23). However, in the scar formation phase, molecular analysis has been scant, except in respect to TGF $\beta$. To answer two important questions for both cardiologists and basic scientists who are interested in pathological myocardial healing, i.e., "what regulates formation of 
the scar phase of an ischemic injury?" and "what is the nature of the factors responsible for the ventricular healing process after AMI?," we focused on periostin, which is a TGF $\beta$-responding factor (1).

\section{RESULTS AND DISCUSSION}

To assess the importance of periostin in the cardiac healing process, we examined the expression of human periostin protein in the myocardial tissue of the left ventricle (LV). No expression of it was observed in the normal myocardium (Fig. $1 \mathrm{~A}$ ), whereas immunoreactivity indicating periostin was detected in Azan-stained myocardial fibrous areas from a patient with AMI (Fig. 1, B and C), thus suggesting that periostin expression was induced in the infarct regions after AMI. In the fibrous area, strong immunoreactivity of periostin was observed around cardiac fibroblasts expressing $\alpha \mathrm{v}$-integrin, which is reported to be a receptor for periostin (Fig. 1 C) $(2,6)$. Next, we examined the expression of periostin in mice after AMI caused by left anterior descending artery (LAD) ligation (24). Periostin protein was not observed up to day 2 , but became detectable at day 3 in the areas showing inflammatory infiltration (Fig. 1 D). This expression in the infarct LV increased significantly at day 4, and was still present at day 28 (Fig. $1 \mathrm{D}$ and not depicted). To identify the cells producing periostin, we performed RNA in situ hybridization to detect periostin mRNA in the infarct LV wall of mice. Periostin mRNA was mainly expressed in fibroblasts in both the infarct and noninfarct regions after AMI (Fig. 1 E). To confirm the periostin expression in cardiac fibroblasts, we performed RT-PCR analysis on purified cardiac cells, and these results showed the expression to be mainly in cardiac fibroblasts, but not in cardiomyocytes (Fig. S1, available at http://www.jem.org/cgi/content/full/jem.20071297/DC1). Furthermore, these fibroblasts were positive for $\alpha v$-integrin, as indicated by flow cytometry using cultured cardiac cells (Fig. S1). The mRNA of $\beta i g-h 3$, another fasciclin family member, which is also expressed in the embryonic heart (25), was not observed in

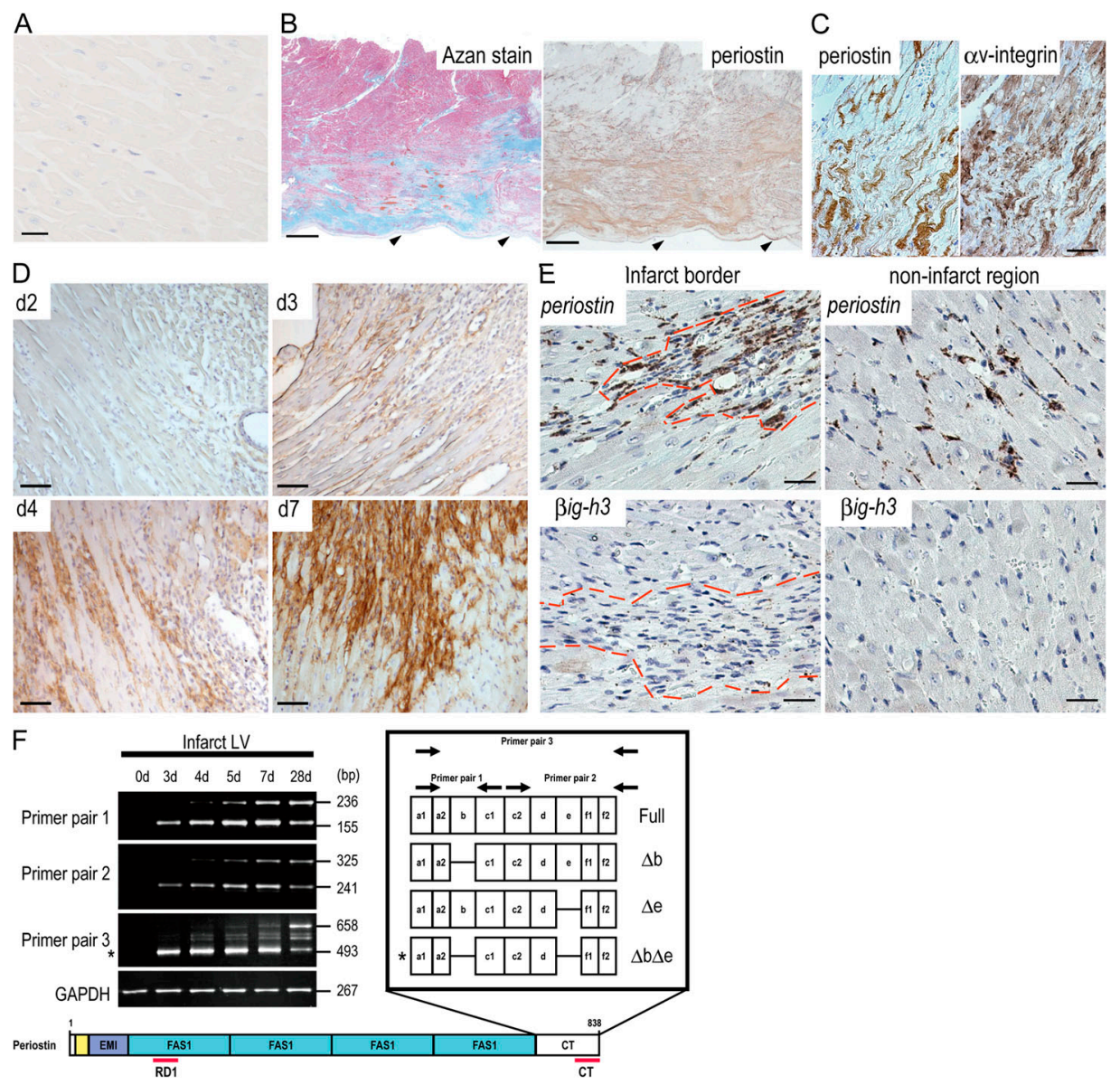

Figure 1. Periostin expression is induced after myocardial ischemia. (A-C) Detection of periostin in myocardium from human patients. LV tissue from a patient with alcoholic cirrhosis $(A)$ and from a patient with $A M I(B$ and $C)$. As seen by immunostaining, periostin protein was detected ( $B$, right) in the myocardial area, which was shown to be fibrous by Azan staining ( $\mathrm{B}$, left). Arrowheads in $\mathrm{B}$ indicate endocardium. (C) Comparison of the expression pattern between periostin (left) and $\alpha \mathrm{V}$-integrin (right) in the fibrous area. (D-F) Periostin is up-regulated after AMI in mice. (D) Immunostaining of periostin after AMI. (E) Expression of periostin (top) and $\beta$ ig- $h 3 \mathrm{mRNA}$ (bottom) in the infarct LV wall of mice was analyzed by in situ hybridization. The dashed red line shows the infarct border. (F) Expression of spliced variant forms of periostin at various times after AMI. Periostin $\Delta b \Delta \mathrm{e}$ is indicated by the asterisk. Bars: (A) $25 \mu \mathrm{m}$; (B) 2 mm; (C-E) $50 \mu \mathrm{m}$. 
the same regions (Fig. $1 \mathrm{E}$ ), thus suggesting the AMI-induced expression of fasciclin family molecules to be specific to periostin.

Because we previously reported that several periostin transcripts exist in human and mouse, caused by alternative splicing at a $3^{\prime}$ site (1), we examined the expression of the splice variants in a time course experiment by RT-PCR analysis using three combinations of specific primers (Fig. $1 \mathrm{~F}$ ). We observed four different isoforms, i.e., $\Delta \mathrm{b}$ (deletion of $\mathrm{b}$ domain), $\Delta \mathrm{e}$ (deletion of e domain), $\Delta \mathrm{b} \Delta \mathrm{e}$ (deletion of $\mathrm{b}$ and e domains), and Full (full-length), and we found that the pattern of splicing depended on the time after AMI. Interestingly, one specific spliced form, $\Delta \mathrm{b} \Delta \mathrm{e}$ (Fig. $1 \mathrm{~F}$, asterisk), was dominantly found as the lowest electrophoretic band in the initial stages $(3,4$, and $5 \mathrm{~d}$ after AMI), indicating the involvement of $\Delta \mathrm{b} \Delta \mathrm{e}$ periostin in the early healing stage of damaged tissues. By $28 \mathrm{~d}$, all 4 isoforms were equally expressed. We also confirmed the expression of these isoforms at the protein level, and found the proteolytic modification of periostin during infarct healing (Fig. S1).

To investigate the role of periostin in AMI, we generated periostin ${ }^{-1-}$ mice combined with Cre recombination (Fig. $2 \mathrm{~A}$ and Fig. S2, available at http://www.jem.org/cgi/content/ full/jem.20071297/DC1). The embryogenesis of periostin ${ }^{-/-}$ mice was apparently normal; and after the birth, the mice appeared to be healthy. The observation of periostin in the developing heart prompted us to thoroughly investigate the heart structure and function in the periostin ${ }^{-/-}$mice; however, no cardiomyocyte abnormalities were found in the myocardium, valve function, pulsation, or blood pressure in the 10-wk-old mice (Fig. S2 and not depicted), which is consistent with no significant expression in the adult myocardium. We then subjected periostin ${ }^{-/-}$mice to AMI by LAD ligation. There was no significant difference in body weight or heart rate among ${ }^{-/-},{ }^{+/-}$, and ${ }^{+/+}$in the normal control condition or after the AMI (Fig. S2, Table S1, and not depicted); moreover, there was no difference in infarct size between the periostin ${ }^{+/+}$and ${ }^{-/-}$mice after AMI (Table S1). However, the survival rate of periostin ${ }^{-1-}$ mice after AMI was significantly lower than that of $^{+/+}$mice (17.58 vs. $53.76 \%$ at day $10 ; \mathrm{P}<0.0001$; Fig. 2 B), whereas this rate of periosti ${ }^{+/-}$mice $(55 \%)$ after AMI was similar to that of ${ }^{+/+}$mice. The incidence of mortality in periostin ${ }^{-/-}$ mice, mainly caused by cardiac rupture, which occurred within $7 \mathrm{~d}$, was significantly higher $(\mathrm{P}<0.001)$ than that of ${ }^{+/+}$mice: 62/91 (68.1\%) in ${ }^{-/-}$versus 25/80 (31.3\%) in ${ }^{+/+}$(Fig. 3 C), whereas this frequency of ${ }^{+/-}$mice $6 / 20(30 \%)$ was similar to that of ${ }^{+/+}$mice. Thereafter, these survival rates reached a plateau from $8 \mathrm{~d}$ up to $4 \mathrm{wk}$ after AMI (unpublished data). To test whether the increased rate of cardiac rupture was caused by abnormal LV wall stiffness, we analyzed the rupture threshold stiffness of the LVs of periostin ${ }^{-/-}$and ${ }^{+/+}$mice $4 \mathrm{~d}$ after AMI by conducting an LV distending pressure/rupture threshold study (18). Myocardial tearing was found at the infarct border in all the ruptured LVs, and the mean of the maximum rupture pressure was significantly lower in periostin ${ }^{-1-}$ mice than in $^{+/+}$mice after AMI $\left(312.7 \pm 3.2 \mathrm{mmHg}\right.$ in ${ }^{-/-}$vs. $374.3 \pm$ $5.8 \mathrm{mmHg}$ in $\left.{ }^{+/+} ; \mathrm{P}=0.0008 ; n=5\right)$, and the mean passive stiffness was also significantly lower in ${ }^{-/-}$mice than in ${ }^{+/+}$mice after AMI $\left(50.26 \pm 2.13 \mathrm{mmHg} / 100 \mu \mathrm{l}\right.$ in ${ }^{-/-}$vs. $65.08 \pm 2.55$ $\mathrm{mmHg} / 100 \mu$ in $^{+/+} ; \mathrm{P}=0.001 ; n=5$; Fig. 2 C). In contrast, no significant difference was observed between ${ }^{+/+}$control noninfarct mice and periostin ${ }^{-1-}$ control noninfarct mice (maximum rupture pressure was $544.0 \pm 6.93 \mathrm{mmHg}$ in ${ }^{-/-}$vs. $552.7 \pm 7.86 \mathrm{mmHg}$ in ${ }^{+/+} ; \mathrm{P}=0.4546 ; n=5$; mean passive stiffness was $87.07 \pm 4.41 \mathrm{mmHg} / 100 \mu \mathrm{l}$ in $^{-/-}$vs. $88.85 \pm$ $\left.3.14 \mathrm{mmHg} / 100 \mu \mathrm{lin}{ }^{+/+} ; \mathrm{P}=0.5985 ; n=5\right)$. These biomechanical data indicate that both rupture threshold and passive stiffness in the LV of the periostin ${ }^{-1-}$ infarcted mice were significantly lower than those of the ${ }^{+/+}$mice after AMI, suggesting that the periostin ${ }^{-/-}$infarct LV wall was more susceptible to cardiac rupture by mechanical stress. Although periostin deficiency did not affect heart structure, the circulatory system, or cardiac performance under physiological conditions, periostin induced in the infarct myocardium appears to play a pivotal role in the healing process after AMI.

To confirm the histomorphological stiffness of the wall in periostin ${ }^{-/-}$mice just escaping from rupture, we performed echocardiography $7 \mathrm{~d}$ after AMI, in addition to $1 \mathrm{~d}$ for heart tissue evaluation and $28 \mathrm{~d}$ for the analysis of chronic cardiac pathophysiology after AMI (Fig. 2 D and Table S1). Echocardiographic measurements made $7 \mathrm{~d}$ after AMI showed decreases in left ventricular end-diastolic dimension (LVEDD) and left ventricular end-systolic dimension (LVESD) in periostin $^{-/-}$mice $(n=10)$, as compared with these parameters for ${ }^{+/+}$mice $\left(n=15\right.$; LVEDD and LVESD values for ${ }^{-/-}$were 89.0 and $84.4 \%$, respectively, of those for $^{+/+}$). These results demonstrate that the absence of periostin attenuated ventricular remodeling after AMI. To further examine tissue stiffness histologically, we performed toluidine blue staining, immunofluorescence analysis using anti-collagen I, -fibronectin, and -vimentin antibodies, and transmission electron microscopic (TEM) observation of sections prepared from periostin ${ }^{+/+}$and ${ }^{-1-}$ mice $5 \mathrm{~d}$ after AMI. The results showed a lower number of cardiac fibroblasts, along with sparser pericellular ECM density in the periostin ${ }^{-/-}$mice than in the ${ }^{+/+}$mice (Fig. 2, E and F); indeed, the number of vimentin-positive cardiac fibroblasts was decreased in the infarct region of periostin ${ }^{-1-}$ mice $5 \mathrm{~d}$ after AMI $\left(7,655 \pm 148\right.$ cells $/ \mathrm{mm}^{2}$ in ${ }^{+/+}$vs. $6,913 \pm 297$ cells/ $\mathrm{mm}^{2}$ in ${ }^{-1-} ; n=6$; $\mathrm{P}<0.02$; Fig. $2 \mathrm{C}$ ). Furthermore, reduced collagen I and fibronectin immunoreactivity was observed in the infarct border of the ${ }^{-/-}$mice (Fig. $2 \mathrm{~F}$ and Fig. S3, available at http://www.jem.org/cgi/content/full/jem.20071297/DC1), and the collagen fiber cross-sectional area (CSA) in the infarct border of periostin ${ }^{-1-}$ mice was significantly smaller and more uniform than that of ${ }^{+/+}$mice $5 \mathrm{~d}$ after AMI (CSA of $1,014.642 \pm 17.546 \mathrm{~nm}^{2}$ for the ${ }^{-/-}$and 2,233.780 \pm 25.731 $\mathrm{nm}^{2}$ for the ${ }^{+/+} ; n=6 ; \mathrm{P}<0.001$, respectively; Fig. $\left.2 \mathrm{G}\right)$. To confirm whether periostin deficiency affected the biochemical property of collagen after AMI, we evaluated the amount of collagen (hydroxyproline concentration, percentage of tissue dry weight) and nonreducible mature cross-links (mol pyridinoline per mol collagen) in the infarct zone $4 \mathrm{~d}$ after AMI. We detected a significant decrease in the collagen crosslinking in the periostin ${ }^{-/-}$mice, compared with the ${ }^{+/+}$mice 
A
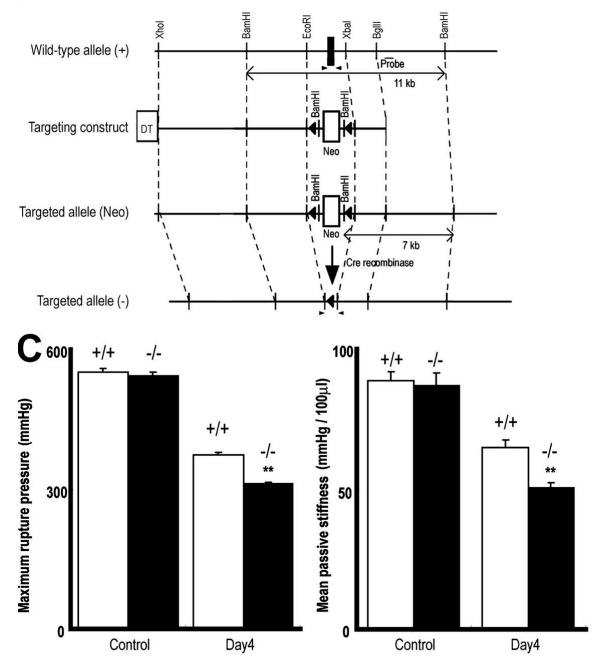

E
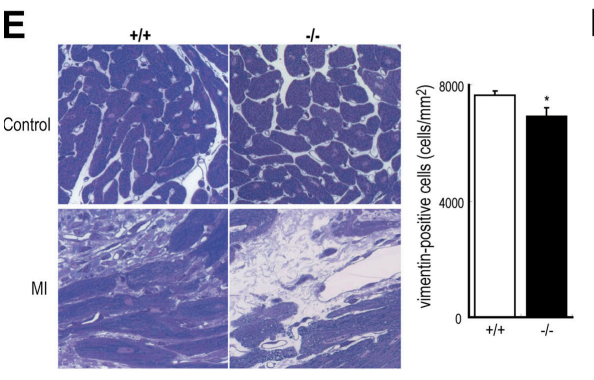
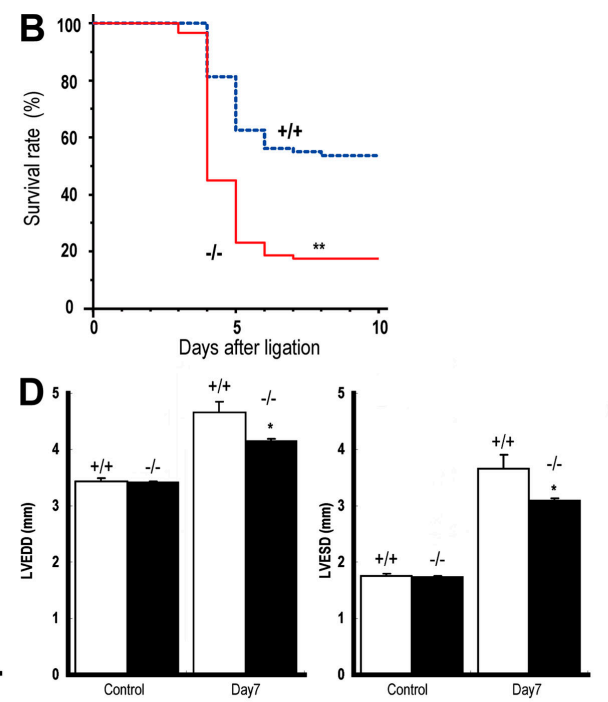

$\mathbf{F}$
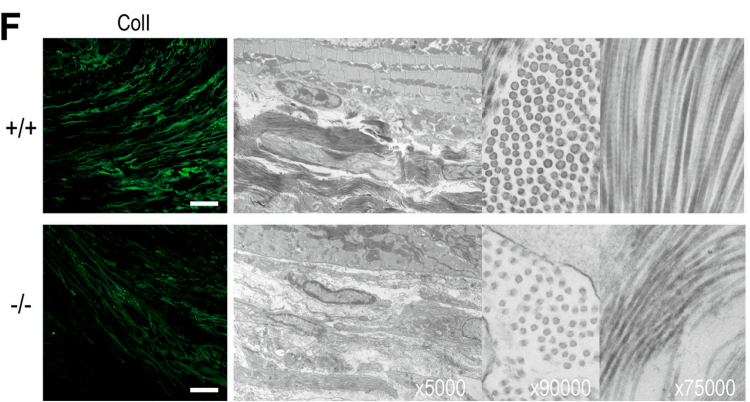

I
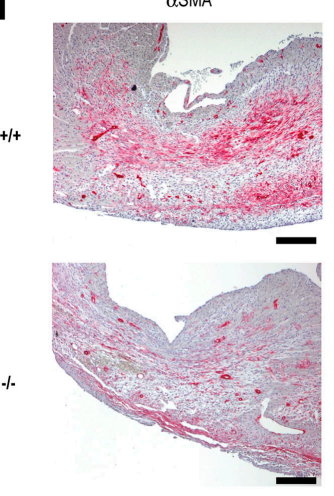

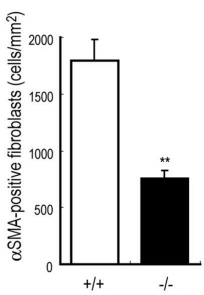

Figure 2. Cardiac rupture after AMI is caused by periostin disruption. (A) Schema of the targeting strategy deletes the first exon of periostin locus. (B) Decreased survival of periostin ${ }^{-1-}$ mice $(n=91)$ compared with the survival of $+1+$ mice $(n=80)$ after AMI. ${ }^{* *}, P<0.0001$. (C) Infarct LV wall stiffness was more reduced in periostin ${ }^{-1-}$ mice than in ${ }^{+/+}$mice after AMI (left). Mean passive stiffness was also significantly lower in the ${ }^{-1-}$ mice than in the ${ }^{+/+}$mice after AMI (right). Open columns, ${ }^{+++}$; filled columns, ${ }^{-1-}{ }^{* *}, P<0.005$, compared with ${ }^{+/+}$mice. (D) Loss of periostin attenuated cardiac dilation after AMI, as shown by echocardiography. Open columns, ${ }^{++}$; filled columns, ${ }^{-1-} .{ }^{*}, \mathrm{P}<0.05$ compared with ${ }^{+/+}$mice. (E) Histological analysis of heart sections from periostin ${ }^{-1-}$ and ${ }^{+/+}$mice stained with toluidine blue $5 \mathrm{~d}$ after AMI, showing a lower number of cardiac fibroblasts and lower ECM density in ${ }^{-1-}$ mice. (right) The number of vimentin-positive cells. ${ }^{*}, \mathrm{P}<0.02$, compared with ${ }^{++}$mice. (F) Images of the infarct border stained with anti-collagen I (left), and TEM images of infarct border, showing evidence of smaller and less abundant collagen in tissues from periostin ${ }^{-1-}$ mice $5 \mathrm{~d}$ after AMI compared with the collagen of the ${ }^{+/+}$infarct heart. Bar, $50 \mu \mathrm{m}$. (G) CSA distribution of collagen fibrils in the infarct border of ${ }^{+1+}$ and ${ }^{-1-}$ mice, measured from TEM images. (H) Biochemical analysis of the collagen amount and cross-linking. ${ }^{*}, \mathrm{P}<0.05 ;{ }^{* *}, \mathrm{P}<0.01$, compared with ${ }^{+/+}$mice. (I) The number of $\alpha S M A-p o s i t i v e$ cells in the infarct area was reduced in periostin ${ }^{-1-}$ mice $5 \mathrm{~d}$ after AMI. (right) The number of $\alpha$ SMA-positive cells. ${ }^{* *}, \mathrm{P}<0.01$, compared with ${ }^{+l+}$ mice. Error bars represent the mean \pm the SEM. Bars, $200 \mu \mathrm{m}$. 
$\left(1.555 \pm 0.461 \mathrm{in}^{-/-}, n=4\right.$, vs. $6.433 \pm 0.919$ in $^{+/+}, n=7$ $\mathrm{P}=0.0043$; Fig. $2 \mathrm{H})$. Moreover, the periostin ${ }^{-1-}$ infarct LV tissue exhibited $52.9 \%$ less collagen content compared with the ${ }^{+/+}$tissue $\left(7.832 \pm 2.241 \%\right.$ in $^{-/-}, n=4$, vs. $14.795 \pm$ $1.565 \%$ in ${ }^{+/+}, n=7 ; \mathrm{P}=0.0283$; Fig. $\left.2 \mathrm{H}\right)$. In normal heart tissues from mice of either genotype, the collagen amount was under the detection level by our methods (unpublished data), indicating that the detected collagen was newly produced after AMI. In conclusion, we observed the alterations of collagen structure in the periostin ${ }^{-/-}$mice; they were smaller and more uniform, with the decreased amount and cross-linking of collagen effecting lower stiffness. These results suggest that periostin expression contributed significantly to the amount or cross-linking of newly synthesized collagen, which is essential for the normal mechanical properties of collagen-containing tissues after MI. These findings indicate that impaired collagen fiber formation occurred in periostin ${ }^{-1-}$ mice after AMI. Interestingly, although the total activity of myeloperoxidase and the numbers of Mac-3-positive inflammatory cells, ki67positive proliferating cells, and active caspase-3-positive apoptotic cells in the infarct border were not significantly different between ${ }^{+/+}$and ${ }^{-/-}$mice (not depicted), we observed a lower number of $\alpha$ SMA-positive cells in the infarct area of periostin ${ }^{-1-}$ mice $5 \mathrm{~d}$ after AMI $\left(1,792 \pm 193\right.$ cells $/ \mathrm{mm}^{2}$ in $^{+/+}$vs. $758 \pm 75$ cells $/ \mathrm{mm}^{2}$ in ${ }^{-/-}$; $\mathrm{P}<0.01 ; n=6$; Fig. $\left.2 \mathrm{I}\right)$.
However, the number of cells positive for SM1, which is a specific marker of SMCs, was not significantly different, and almost all of the $\alpha$ SMA-positive cells were SM1 negative (unpublished data). These results indicate that not the inflammatory cell recruitment, but rather the recruitment of cardiac fibroblasts in the infarct region, was impaired in these animals.

To determine whether the impaired cardiac healing in response to AMI could be restored by periostin directly, we performed a rescue experiment by using $\Delta \mathrm{b} \Delta \mathrm{e}$, which is the main periostin isoform detected early after AMI. The periostin ${ }^{-1-}$ mice were treated with a recombinant adenovirus expressing periostin $(\mathrm{Ad}-\Delta \mathrm{b} \Delta \mathrm{e}$ ) or with a control adenovirus (Ad-nls; nuclear localization signal-LacZ). In the control experiment, the Ad-nls-LacZ transfer was detected in the infarct border at $4 \mathrm{~d}$ after AMI by whole-mount $\mathrm{X}$-gal staining, proving the experimental feasibility (Fig. 3 A). In periostin ${ }^{-1-}$ mice infected with Ad- $\Delta \mathrm{b} \Delta \mathrm{e}$, we first confirmed expression of transferred periostin in the infarct tissue by immunoblot and immunofluorescence analyses (Fig. $3 \mathrm{~B}$ and Fig. S4, available at http://www.jem.org/cgi/content/full/jem.20071297/DC1), and then observed an increase in the area reactive with anti$\alpha$ SMA antibody compared with that area of the control Ad-nlsLacZ-infected periostin ${ }^{-/-}$mice $\left(597 \pm 107\right.$ cells $/ \mathrm{mm}^{2}$ in Ad-nls-LacZ-infected ${ }^{-/-}$mice vs. $1,535 \pm 197$ cells $/ \mathrm{mm}^{2}$

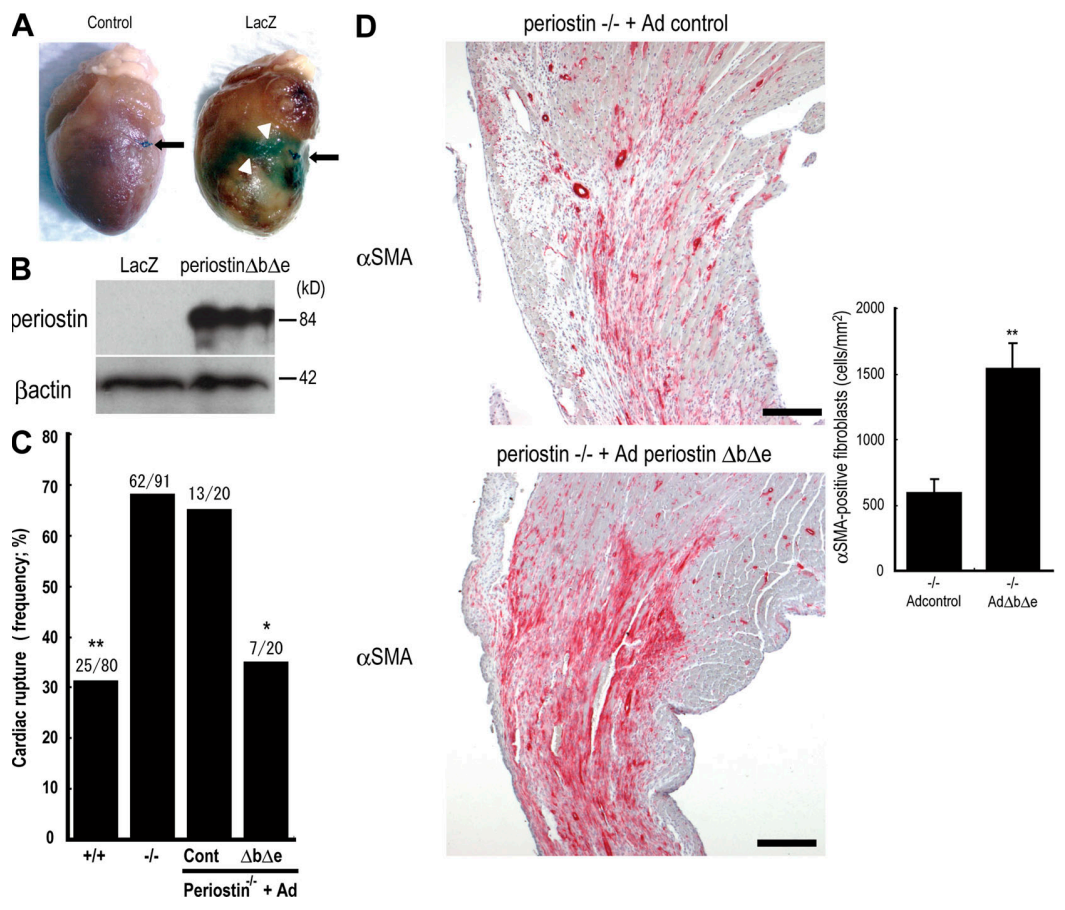

Figure 3. Adenovirus-mediated periostin $\Delta \mathbf{b} \Delta \mathrm{e}$ gene transfer prevents cardiac rupture in the periostin ${ }^{-/-}$mice. (A) Whole-mount X-gal staining $4 \mathrm{~d}$ after AMI showed strong expression in the border of the Ad-nls-LacZ-infected myocardial infarct (arrowheads). The arrow indicates the ligated portion. (B) Western blot analysis for Ad- $\Delta b \Delta \mathrm{e}$-infected periostin ${ }^{-1-}$ infarct LV. (C) Infection with Ad- $\Delta b \Delta$ e reversed the high incidence of cardiac rupture in the periostin ${ }^{-1-}$ mice to a lower level, comparable to the incidence in the ${ }^{+/+}$mice. ${ }^{*}, P<0.02{ }^{*}{ }^{*}, P<0.001$, compared with control Ad-treated ${ }^{-1-}$ mice. (D) Compared with the Ad-nls-LacZ-infected periostin-1- hearts, the Ad- $\Delta b \Delta$ e-infected hearts increased the number of $\alpha$ SMA-positive cells $5 d$ after AMI. (right) the number of $\alpha$ SMA-positive cells. ${ }^{* *}, \mathrm{P}<0.01$, compared with the mock infection of the ${ }^{-1-}$ mice. Error bars represent the mean \pm the SEM. Bars, $200 \mu \mathrm{m}$. 
in $\mathrm{Ad}-\Delta \mathrm{b} \Delta \mathrm{e}^{-}$-infected ${ }^{-/-}$mice; $\mathrm{P}<0.01 ; n=6$; Fig. $\left.3 \mathrm{D}\right)$. Furthermore, the Ad- $\Delta \mathrm{b} \Delta \mathrm{e}$ infection reduced the incidence of rupture frequency in periostin ${ }^{-1-}$ mice $(35.0 \%)$ compared with that for the Ad-nls-LacZ-treated $-/-$ mice $(65.0 \%$; Fig. $3 \mathrm{C}$ ). These results demonstrate that periostin $\Delta \mathrm{b} \Delta \mathrm{e}$ was essential for in vivo recruitment of $\alpha$ SMA-positive fibroblasts to block rupture after AMI. As cell motility and morphology of fibroblasts are associated with the expression of the phosphorylated forms of Akt and focal adhesion kinase (FAK) $(26,27)$, we examined the phosphorylation of these proteins in the infarct border $5 \mathrm{~d}$ after AMI. The amount of phosphorylated
Akt was reduced, and only a small amount of phosphorylated FAK was detected in the border of the periostin ${ }^{-1-}$ infarcted mice (Fig. 4, A and B, and Fig. S5).

To further investigate the role of periostin in FAK activation and cell motility, we performed immunofluorescence staining for phosphorylated-FAK and rhodamine-phalloidin staining for the actin cytoskeleton in an embryonic mesenchymal cell line, C3H10T1/2, treated or not with periostin $\Delta \mathrm{b} \Delta \mathrm{e}$. The presence of periostin $\Delta \mathrm{b} \Delta \mathrm{e}$ changed the cytoskeletal arrangement and motility of the cells, resulting in dynamic protrusion of their processes (Fig. 4 C). In a time-course
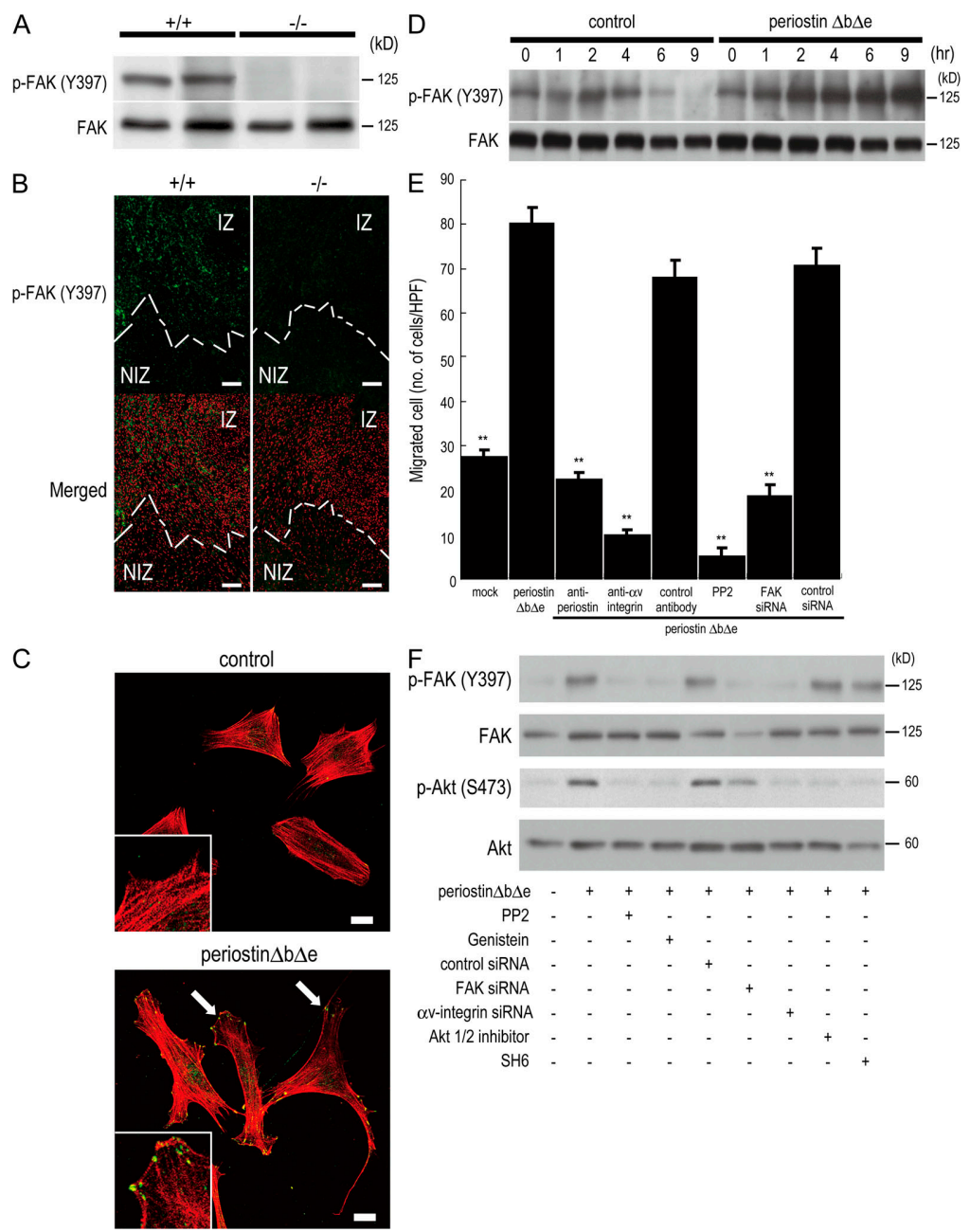

Figure 4. Periostin promotes cell migration through integrin-mediated FAK signaling. (A) Phosphorylation of FAK in infarct LV from periostin ${ }^{+/+}$ mice and ${ }^{-/-}$mice $5 \mathrm{~d}$ after AMI. (B) Immunofluorescence for phosphorylated FAK (p-FAK ${ }^{\mathrm{3} 97}$ ) in the border of infarct $\mathrm{LV}_{\text {from }}$ periostin ${ }^{+/+}$mice and ${ }^{-/-}$ mice $5 \mathrm{~d}$ after AMI. Merged images show an overlay of $\mathrm{p}-\mathrm{FAK}^{\Upsilon 397}$ (green) and propidium iodide-stained nuclei (red). The dotted line shows the infarct border. NIZ, noninfarct zone; IZ, infarct zone. (C and D) Promotion of cell spreading and activation of FAK phosphorylation in vitro. The morphology of starved $\mathrm{C} 3 \mathrm{H} 10 \mathrm{~T} 1 / 2$ cells was analyzed by immunofluorescence $12 \mathrm{~h}$ after adding periostin $\Delta \mathrm{b} \Delta \mathrm{e}$ (C), and the p-FAK ${ }^{\Upsilon 397}$ was examined by Western blot analysis at various times after adding periostin $\Delta b \Delta \mathrm{e}(\mathrm{D})$. In $\mathrm{C}$, the merged images show an overlay of $\mathrm{p}$-FAK $\mathrm{F}^{\mathrm{Y} 97}$ (green) and rhodamine-phalloidin (red), and the arrows point to FAK phosphorylation sites. The insets show higher magnification of the cell processes. (E) Chemotaxis of primary cardiac fibroblasts from periostin ${ }^{-1-}$ mice in the absence (mock) or presence of periostin $\Delta \mathrm{b} \Delta \mathrm{e}$, detected by an in vitro cell migration assay. Cardiac fibroblasts were significantly activated by periostin $\Delta \mathrm{b} \Delta \mathrm{e}$, and treatment with neutralizing antibodies against periostin and $\alpha \mathrm{V}$-integrin, PP2, or FAK siRNAs reduced the cell migration. ${ }^{*}, \mathrm{P}<0.001$ vs. periostin $\Delta \mathrm{b} \Delta$ e. Error bars represent the mean \pm the SEM. (F) Periostin can stimulate FAK and Akt phosphorylation through integrin signaling. Starved C3H10T1/2 cells were incubated for $1 \mathrm{~h}$ with periostin $\Delta \mathrm{b} \Delta \mathrm{e}$ with or without each siRNA or the FAK and Akt inhibitors. Bars: (B) $100 \mu \mathrm{m}$; (C) $20 \mu \mathrm{m}$. 
experiment, periostin $\Delta \mathrm{b} \Delta \mathrm{e}$ continuously activated the phosphorylation of FAK for $9 \mathrm{~h}$ after the addition of it to serumstarved cell cultures, whereas in the control, the signal had decreased by $6 \mathrm{~h}$ (Fig. $4 \mathrm{D}$ ). These results demonstrate that periostin $\Delta \mathrm{b} \Delta \mathrm{e}$ activated FAK phosphorylation and promoted formation of dynamic protrusions. Next, we tested the motility of primary cardiac fibroblasts from periostin ${ }^{-/-}$mice in the presence of periostin $\Delta \mathrm{b} \Delta \mathrm{e}$. The result showed that periostin $\Delta \mathrm{b} \Delta \mathrm{e}$ strongly activated the cell migration of these fibroblasts (Fig. 4 E). Moreover, this migration caused by periostin $\Delta \mathrm{b} \Delta \mathrm{e}$ was significantly reduced by antibodies against either periostin or $\alpha \mathrm{v}$-integrin; by PP2, which is known as a compound that specifically inhibits adhesion-induced FAK phosphorylation (28); and by knockdown of FAK by siRNA (Fig. 4 E), suggesting that periostin $\Delta \mathrm{b} \Delta \mathrm{e}$ would activate the cell motility of their fibroblasts by FAK signaling through $\alpha v$-integrin in mice subjected to AMI. Finally, we inhibited the integrin-mediated FAK pathway by using chemical compounds and siRNAs (Fig. 4 F). FAK inhibitors or siRNA down-regulated the Akt phosphorylation, and Akt inhibitors did not change FAK phosphorylation after stimulation by periostin $\Delta \mathrm{b} \Delta \mathrm{e}$, indicating that Akt is a downstream molecule of FAK and periostin $\Delta \mathrm{b} \Delta \mathrm{e}$. Moreover, $\alpha \mathrm{v}$-integrin siRNA treatment blocked both FAK and Akt phosphorylation after stimulation by periostin $\Delta \mathrm{b} \Delta \mathrm{e}$. These results indicate that periostin $\Delta \mathrm{b} \Delta \mathrm{e}$ can stimulate FAK and Akt phosphorylation through $\alpha \mathrm{v}$-integrin.

We demonstrated that in the case of periostin deficiency, the collagen amount was reduced in the infarct myocardium, resulting in frequent cardiac rupture in the AMI. Our results, together with the previous findings by Norris et al. (7) on the role of periostin in collagen fibrillogenesis of skin and tendon, strongly suggest that fibrillar collagen formation, which contributes essentially to a mechanically stable scar formation, was impaired in the early stage of MI in the periostin deficiency, resulting in a high rate of cardiac rupture. Furthermore, we have found that the reduced mechanical strength, rupture of the infarct region, and repression of LV dilation in periostin deficiency were most likely caused by a reduced number of cardiac fibroblasts and by the insufficient creation of a durable collagen network caused by a lower rate of collagen synthesis and cross-linking. To reveal more about the importance of collagen production or collagen cross-linking for protection against heart rupture, after AMI, we treated mice with an inhibitor of lysyl oxidase, thus inhibiting collagen cross-linking. Interestingly, the data showed a high amount of collagen production with a larger number of vimentin-positive cells in the infarct region, resulting in effective blockage of heart rupture (unpublished data). These data suggest that periostin-stimulated migration of cardiac fibroblasts into the infarct region, the cells of which produce a high amount of collagen, is more essential than collagen cross-linking by periostin.

The expression of TGF $\beta$ was markedly up-regulated in the infarct border during the scar formation phase after AMI, and the phosphorylation of smad $2 / 3$ was consequently increased (unpublished data), whereas there was no significant difference in the TGF $\beta$ transcription level between periostin ${ }^{+/+}$and
-I- mice; TGF $\beta$ also enhanced the periostin expression in the infarct border after AMI because anti-TGF $\beta$ antibody treatment blocked the periostin expression (Fig. S6, available at http://www.jem.org/cgi/content/full/jem.20071297/DC1). The expression of both TGF $\beta$ and periostin is up-regulated by angiotensin II and attenuated by angiotensin receptor blockers after AMI $(29,30)$, suggesting that periostin may play a role via angiotensin II-TGF $\beta$ signaling. The combined results on the biomechanical properties and the collagen content of the isolated infarct heart support the concept that the periostinlinked collagen fibrous skeleton is an important determinant of cardiac rupture.

The results given here indicate that periostin signals activate cell migration of cardiac fibroblasts from outside into the infarct region through FAK phosphorylation, and then the migrated cells differentiate into $\alpha$ SMA-positive fibroblasts, resulting in strengthening of the stiffness of the LV wall through collagen synthesis after AMI. FAK is known to be involved in tyrosine phosphorylation during integrin-mediated signaling, and this molecule plays an important role in the response of migrating cells to mechanical stress (31). Recently, FAK has been implicated as a downstream target associated with angiotensin II-stimulated cell migration (32). The mechanism underlying the periostin action of promoting the recruitment of cardiac fibroblasts followed by healing of the infarct region appears to involve activation of the FAK pathway, indicating that the periostin-induced increase in FAK phosphorylation in the infarct myocardium enhanced the motility of these fibroblasts. In contrast, three-dimensional culture studies imply that the matrix stiffness regulates cell fate by modulating integrin signaling $(31,33)$. Considering these accumulated results, we suggest that periostin is mainly produced by fibroblasts through angiotensin II-TGF $\beta$ signaling and may convey pathologically rapid reinforced mechanical signals to FAK-integrin signaling after AMI. The fibroblastic cells activated by these signals secrete periostin, which in turn increases their motility, contractility, and synthesis of ECM proteins, thus promoting further recruitment and activation of fibroblasts. Periostin may serve as the trigger of these feedback mechanisms in the ongoing healing processes. Additional studies to elucidate in more detail the characteristics of cardiac fibroblasts may lead to a deeper understanding of the role of periostin after AMI, as well as aid in identifying the molecular targets of therapies to augment cardiac performance and wall stiffness after AMI.

\section{MATERIALS AND METHODS}

Preparation of rabbit polyclonal antibodies against periostin. We raised polyclonal RD1 antibodies against periostin by using the peptide DNLDSDIRR GLESNVN (representing a 143-158 of human periostin) for human periostin and the peptide ENLDSDIRRGLENNVN (representing aa 145-160 of mouse periostin) for mouse periostin. The antibodies were affinity-purified by using the respective immunogenic peptide.

Histology, immunostaining, and electron microscopy. Human tissue samples were obtained during autopsy and fixed in 4\% neutral formalin or $20 \%$ formalin. A total of 41 cases, ranging from a fetus to an 89-yr-old patient, including 15 cases of myocardial infarction, were examined. All the cases were approved for use in research by the Ethics Committee of the 
University of Tokyo. After having been embedded in paraffin, specimens were cut at a $4-\mu \mathrm{m}$ thickness. Hematoxylin and eosin, elastica von Gieson, and Azan staining procedures were performed. Immunohistochemistry by the $\mathrm{ABC}$ method was done by using an 16000 apparatus (Biogenex).

For histological analysis of the infarcted mice, the animals were killed at $1,2,3,4,5,7,14$, or $28 \mathrm{~d}$ after surgery under anesthesia, and were perfusion fixed with $4 \%$ paraformaldehyde at physiological pressure. Fixed hearts were sectioned transversely into three equal segments from their apex to base and cryoembedded or embedded in paraffin. 4- $\mu$ m-thick sections were used for histological analysis or for immunostaining. Antibodies against $\alpha \mathrm{V}$-integrin (Laboratory Vision), $\alpha$ SMA (Sigma-Aldrich), FAK (BD Biosciences), pY397FAK (Invitrogen), pS473Akt (Cell Signaling Technology), Akt (Cell Signaling Technology), collagen I (Novotec), fibronectin (34), Ki67 (YLEM), active caspase3 (Promega), vimentin (PROGEN), smooth muscle myosin-1 (SM1; Kyowa Hakko Ltd.), and Mac3 (BD Biosciences) were used for immunostaining. Antigen unmasking techniques were not performed, except for anti- $\alpha v$-integrin. For immunostaining of pY397FAK and pS473Akt, the Catalyzed Signal Amplification system was used (Dako). In the case of fluorescence studies, the signals were observed under a confocal microscope (FLUOVIEW FV1000; Olympus).

Sections of infarcted heart were generated from 6 periostin ${ }^{+/+}$and $6^{-1-}$ male mice at 5 and $28 \mathrm{~d}$ after AMI, and they were prepared for electron microscopy as previously described (35). Sham-operated mice were used for the control. Collagen fibril diameters were measured in scanned images generated from electron micrographs with Image $\mathrm{J}$ software. Collagen fibrils and the number of vimentin-positive or $\alpha \mathrm{SMA}$-positive cells in at least 6 fields derived from each of the basement-, mid-, and apex-part of the infarct region of heart sections were quantified (6 mice per group). Animal studies were conducted under a protocol approved by the Institutional Animal Use and Care Committee.

Quantification of collagen cross-links and collagen contents. Snapfrozen infarct tissues from periostin ${ }^{+/+}$and ${ }^{-/-}$mice were used. Pyridinoline and hydroxyproline contents were determined by the previously described HPLC method (36).

Adenovirus-mediated gene transfer. Construction of Ad-nlsLacZ and Ad-periostin $\Delta \mathrm{b} \Delta \mathrm{e}$ vectors was performed by use of an Adeno-X Expression System 2 (BD Biosciences). The virus purification method used, involving cesium chloride ultracentrifugation, was previously described (37). $1 \mathrm{~d}$ before LAD ligation, a volume of $100 \mu \mathrm{l}$ containing $1.6 \times 10^{10} \mathrm{PFU}$ of Ad-nlsLacZ or Ad-periostin $\Delta \mathrm{b} \Delta \mathrm{e}$ virus was injected into a tail vein of male periostin $^{-1-}$ mice.

Statistical analysis. All numerical results were presented as the mean \pm the SEM. Statistical analyses of the echocardiography and cell migration assay were performed with a Student's unpaired $t$ test. Cardiac rupture frequency was compared by the $\chi^{2}$ test. Survival curves after AMI were obtained by the Kaplan-Meier method, and compared by the log-rank test. Differences were considered significant at $\mathrm{P}<0.05$.

Online supplemental material. Fig. S1 shows the confirmation of the periostin expression in cardiac fibroblasts. Fig. S2 indicates the generation of periostin $^{-1-}$ mice. Fig. S3 shows the immunofluorescence analysis of fibronectin after AMI. Fig. S4 shows immunofluorescence analysis for genetransferred periostin ${ }^{-1-}$ infarct heart. Fig. S5 depicts the analysis for the phosphorylation of Akt after AMI. Fig. S6 shows a cause-and-effect relationship between TGF $\beta$ and periostin after AMI. Table S1 provides the echocardiographic data. Full methods and associated references are available in the Supplemental materials and methods. The online version of this article is available at http://www.jem.org/cgi/content/full/jem.20071297/DC1.

The authors thank M. Ikumi and E. Ikeno for technical assistance in generating knockout mice; S. Matsumura and M. Yoshioka for their technical help in the LV rupture threshold study; and S. Sakaguchi for her support, patience, and comprehension.
This research was supported by grants-in-aid from the Ministry of Education, Culture, Sports, Science, and Technology of Japan to A. Kudo and Y. Saga.

The authors have no conflicting financial interests.

Submitted: 25 June 2007

Accepted: 17 December 2007

\section{REFERENCES}

1. Horiuchi, K., N. Amizuka, S. Takeshita, H. Takamatsu, M. Katsuura, H. Ozawa, Y. Toyama, L.F. Bonewald, and A. Kudo. 1999. Identification and characterization of a novel protein, periostin, with restricted expression to periosteum and periodontal ligament and increased expression by transforming growth factor beta. J. Bone Miner. Res. 14:1239-1249.

2. Gillan, L., D. Matei, D.A. Fishman, C.S. Gerbin, B.Y. Karlan, and D.D. Chang. 2002. Periostin secreted by epithelial ovarian carcinoma is a ligand for alpha(V)beta(3) and alpha(V)beta(5) integrins and promotes cell motility. Cancer Res. 62:5358-5364.

3. Bao, S., G. Ouyang, X. Bai, Z. Huang, C. Ma, M. Liu, R. Shao, R.M. Anderson, J.N. Rich, and X.F. Wang. 2004. Periostin potently promotes metastatic growth of colon cancer by augmenting cell survival via the Akt/PKB pathway. Cancer Cell. 5:329-339.

4. Lindner, V., Q. Wang, B.A. Conley, R.E. Friesel, and C.P.H. Vary. 2005. Vascular injury induces expression of periostin: implications for vascular cell differentiation and migration. Arterioscler. Thromb. Vasc. Biol. 25:77-83.

5. Li, G., S. Oparil, J.M. Sanders, L. Zhang, M. Dai, L.B. Chen, S.J. Conway, C.A. McNamara, and I.J. Sarembock. 2006. Phosphatidylinositol-3kinase signaling mediates vascular smooth muscle cell expression of periostin in vivo and in vitro. Atherosclerosis. 188:292-300.

6. Butcher, J.T., R.A. Norris, S. Hoffman, C.H. Mjaatvedt, and R.R. Markwald. 2007. Periostin promotes atrioventricular mesenchyme matrix invasion and remodeling mediated by integrin signaling through Rho/PI 3-kinase. Dev. Biol. 302:256-266.

7. Norris, R.A., B. Damon, V. Mironov, V. Kasyanov, A. Ramamurthi, R. Moreno-Rodriguez, T. Trusk, J.D. Potts, R.L. Goodwin, J. Davis, et al. 2007. Periostin regulates collagen fibrillogenesis and the biomechanical properties of connective tissues. J. Cell. Biochem. 101:695-711.

8. Kruzynska-Frejtag, A., M. Machnicki, R. Rogers, R.R. Markwald, and S.J. Conway. 2001. Periostin (an osteoblast-specific factor) is expressed within the embryonic mouse heart during valve formation. Mech. Dev. 103:183-188.

9. Stanton, L.W., L.J. Garrard, D. Damm, B.L. Garrick, A. Lam, A.M Kapoun, Q. Zheng, A.A. Protter, G.F. Schreiner, and R.T. White. 2000 Altered patterns of gene expression in response to myocardial infarction. Circ. Res. 86:939-945.

10. Urasawa, K., I. Yoshida, C. Takagi, H. Onozuka, T. Mikami, H. Kawaguchi, and A. Kitabatake. 1996. Enhanced expression of betaadrenergic receptor kinase 1 in the hearts of cardiomyopathic Syrian hamsters, BIO53.58. Biochem. Biophys. Res. Commun. 219:26-30.

11. Wang, D., S. Oparil, J.A. Feng, P. Li, G. Perry, L.B. Chen, M. Dai, S.W. John, and Y.F. Chen. 2003. Effects of pressure overload on extracellular matrix expression in the heart of the atrial natriuretic peptidenull mouse. Hypertension. 42:88-95.

12. Katsuragi, N., R. Morishita, N. Nakamura, T. Ochiai, Y. Taniyama, Y Hasegawa, K. Kawashima, Y. Kaneda, T. Ogihara, and K. Sugimura. 2004. Periostin as a novel factor responsible for ventricular dilation. Circulation. 110:1806-1813.

13. Bujak, M., and N.G. Frangogiannis. 2007. The role of TGF-beta signaling in myocardial infarction and cardiac remodeling. Cardiovasc. Res. 74:184-195.

14. Sun, Y., and K.T. Weber. 2000. Infarct scar: a dynamic tissue. Cardiovasc. Res. 46:250-256.

15. Virag, J.I., and C.E. Murry. 2003. Myofibroblast and endothelial cell proliferation during murine myocardial infarct repair. Am. J. Pathol. 163:2433-2440

16. Tomasek, J.J., G. Gabbiani, B. Hinz, C. Chaponnier, and R.A. Brown. 2002. Myofibroblasts and mechano-regulation of connective tissue remodelling. Nat. Rev. Mol. Cell Biol. 3:349-363.

17. Heymans, S., A. Luttun, D. Nuyens, G. Theilmeier, E. Creemers, L. Moons, G.D. Dyspersin, J.P.M. Cleutjens, M. Shipley, A. Angellilo, 
et al. 1999. Inhibition of plasminogen activators or matrix metalloproteinases prevents cardiac rupture but impairs therapeutic angiogenesis and causes cardiac failure. Nat. Med. 5:1135-1142.

18. Matsumura, S.-I., S. Iwanaga, S. Mochizuki, H. Okamoto, S. Ogawa, and Y. Okada. 2005. Targeted deletion or pharmacological inhibition of MMP-2 prevents cardiac rupture after myocardial infarction in mice. J. Clin. Invest. 115:599-609.

19. Matsusaka, H., T. Ide, S. Matsushima, M. Ikeuchi, T. Kubota, K Sunagawa, S. Kinugawa, and H. Tsutsui. 2006. Targeted deletion of p53 prevents cardiac rupture after myocardial infarction in mice. Cardiovasc. Res. 70:457-465.

20. Nahrendorf, M., K. Hu, S. Frantz, F.A. Jaffer, C.-H. Tung, K.-H Hiller, S. Voll, P. Nordbeck, D. Sosnovik, S. Gattenlohner, et al. 2006. Factor XIII deficiency causes cardiac rupture, impairs wound healing, and aggravates cardiac remodeling in mice with myocardial infarction. Circulation. 113:1196-1202.

21. Askari, A.T., M.-L. Brennan, X. Zhou, J. Drinko, A. Morehead, J.D. Thomas, E.J. Topol, S.L. Hazen, and M.S. Penn. 2003. Myeloperoxidase and plasminogen activator inhibitor 1 play a central role in ventricular remodeling after myocardial infarction. J. Exp. Med. 197:615-624.

22. Sun, M., F. Dawood, W.-H. Wen, M. Chen, I. Dixon, L.A. Kirshenbaum, and P.P. Liu. 2004. Excessive tumor necrosis factor activation after infarction contributes to susceptibility of myocardial rupture and left ventricular dysfunction. Circulation. 110:3221-3228.

23. Ichihara, S., T. Senbonmatsu, E. Price Jr., T. Ichiki, F.A. Gaffney, and T. Inagami. 2002. Targeted deletion of angiotensin II type 2 receptor caused cardiac rupture after acute myocardial infarction. Circulation. 106:2244-2249.

24. Michael, L.H., M.L. Entman, C.J. Hartley, K.A. Youker, J. Zhu, S.R. Hall, H.K. Hawkins, K. Berens, and C.M. Ballantyne. 1995. Myocardial ischemia and reperfusion: a murine model. Am. J. Physiol. 269:H2147-H2154.

25. Lindsley, A., W. Li, J. Wang, N. Maeda, R. Rogers, and S.J. Conway. 2005. Comparison of the four mouse fasciclin-containing genes expression patterns during valvuloseptal morphogenesis. Gene Expr. Patterns. 5:593-600.
26. Stambolic, V., and J.R. Woodgett. 2006. Functional distinctions of protein kinase $\mathrm{B} / \mathrm{Akt}$ isoforms defined by their influence on cell migration. Trends Cell Biol. 16:461-466.

27. Mitra, S.K., D.A. Hanson, and D.D. Schlaepfer. 2005. Focal adhesion kinase: in command and control of cell motility. Nat. Rev. Mol. Cell Biol. 6:56-68.

28. Hakuno, D., T. Takahashi, J. Lammerding, and R.T. Lee. 2005. Focal adhesion kinase signaling regulates cardiogenesis of embryonic stem cells. J. Biol. Chem. 280:39534-39544.

29. Iekushi, K., Y. Taniyama, J. Azuma, N. Katsuragi, N. Dosaka, F. Sanada, N. Koibuchi, K. Nagao, T. Ogihara, and R. Morishita. 2007. Novel mechanisms of valsartan on the treatment of acute myocardial infarction through inhibition of the antiadhesion molecule periostin. Hypertension. 49:1409-1414.

30. Berk, B.C., K. Fujiwara, and S. Lehoux. 2007. ECM remodeling in hypertensive heart disease. J. Clin. Invest. 117:568-575.

31. Wang, H.-B., M. Dembo, S.K. Hanks, and Y.-1. Wang. 2001. Focal adhesion kinase is involved in mechanosensing during fibroblast migration. Proc. Natl. Acad. Sci. USA. 98:11295-11300.

32. Baudino, T.A., W. Carver, W. Giles, and T.K. Borg. 2006. Cardiac fibroblasts: friend or foe? Am. J. Physiol. Heart Circ. Physiol. 291:H1015-H1026.

33. Bershadsky, A.D., N.Q. Balaban, and B. Geiger. 2003. Adhesion-dependent cell mechanosensitivity. Annu. Rev. Cell Dev. Biol. 19:677-695.

34. Arai, S., N. Amizuka, Y. Azuma, S. Takeshita, and A. Kudo. 2003. Osteoclastogenesis-related antigen, a novel molecule on mouse stromal cells, regulates osteoclastogenesis. J. Bone Miner. Res. 18:686-695.

35. Kii, I., N. Amizuka, L. Minqi, S. Kitajima, Y. Saga, and A. Kudo. 2006. Periostin is an extracellular matrix protein required for eruption of incisors in mice. Biochem. Biophys. Res. Commun. 342:766-772.

36. Saito, M., K. Marumo, K. Fujii, and N. Ishioka. 1997. Single-column highperformance liquid chromatographic-fluorescence detection of immature, mature, and senescent cross-links of collagen. Anal. Biochem. 253:26-32.

37. Ugai, H., T. Yamasaki, M. Hirose, K. Inabe, Y. Kujime, M. Terashima, B. Liu, H. Tang, M. Zhao, T. Murata, et al. 2005. Purification of infectious adenovirus in two hours by ultracentrifugation and tangential flow filtration. Biochem. Biophys. Res. Commun. 331:1053-1060. 